$16^{\text {th }}$ International Congress of Metrology, 11007 (2013)

DOI: $10.1051 /$ metrology/201311007

(C) Owned by the authors, published by EDP Sciences, 2013

\title{
Grid-based computational algorithm for accurate AC conductivity measurements
}

\author{
Andrei Pokatilov ${ }^{1,2, a}$, Martin Parker $^{2}$, Toomas Kübarsepp ${ }^{1,2}$, Olev Märtens ${ }^{1}$ and Andrei Kolyshkin ${ }^{3}$ \\ ${ }^{1}$ Tallinn University of Technology, Ehitajate tee 5, 19086 Tallinn, Estonia \\ ${ }^{2}$ Metrosert AS - Central Office for Metrology, Teaduspargi 8, 12618 Tallinn, Estonia \\ ${ }^{3}$ Riga Technical University, 1 Meza street, Riga LV-1048, Latvia
}

\begin{abstract}
A novel computational method is proposed for the accurate determination of the electrical conductivity of non-ferrous metal samples in the wide frequency range. The forward and inverse problems solution have been proposed allowing computation of the AC conductivity value from the electrical impedance change measurements of a thoroughly characterised inductive sensor. The developed method is applicable in the accurate electrical conductivity measurements at frequencies up to $500 \mathrm{kHz}$ with the measurement uncertainty less than $1 \%$ of the measured value.
\end{abstract}

\section{Introduction}

Electrical conductivity measurements are widely used in science and industry for example in examination of processed metal parts and quality control of products.

Several calibration techniques based on DC current [1], AC current [2] and combination of both methods [3] have been developed.

For measuring instruments working under the $\mathrm{AC}$ current at frequencies above $100 \mathrm{kHz}$, a method presented in [3] can be extended to higher frequencies. For the latter case we have developed an enhanced computational algorithm for in-measurement $\mathrm{AC}$ conductivity determination. The computation starts from the forward problem solution based on an analytical expression developed by Dodd and Deeds [4]. After that an impedance grid covering the solution space is generated to be searched for the best convergence between the theoretical values and the measurement result. The conductivity determination is automated by realization of the impedance data acquisition and processing software in same programming environment.

In this paper, we present solutions for the forward and inverse problems required for accurate determination of the $\mathrm{AC}$ conductivity value in the wide frequency range. Also, a good agreement between the conductivity values obtained under the AC and DC currents is demonstrated.

\section{Forward problem solution}

Consider an air core coil located above a conducting halfspace with the conductivity $\sigma$. Let $r_{\text {out }}$ and $r_{i n}$ be the outer and inner radius of the coil, respectively, $h_{1}$ and $h_{2}$ are the distances from the bottom (lift-off) and the top of the coil to the conducting half-space, $h=h_{2}-h_{1}$ is the height of the coil and $N$ be the number of turns. The induced change in impedance of the coil is computed in [4] using the solution of the Maxwell's equations for the case where the vector potential has only one non-zero component. The induced change in impedance is given by the formula

$$
Z_{\text {ind }}^{T}=j \omega \pi \mu_{0} \frac{N^{2}}{\left(r_{\text {out }}-r_{\text {in }}\right)^{2}\left(h_{2}-h_{1}\right)^{2}} Q,
$$

where

$$
Q=\int_{0}^{\infty} \frac{\lambda-q}{\lambda^{6}(\lambda+q)}\left(e^{-\lambda h_{2}}-e^{-\lambda h_{1}}\right)^{2} g^{2}\left(r_{\text {in }}, r_{\text {out }}, \lambda\right) d \lambda .
$$

Here the following notations are used: $q=\sqrt{\lambda^{2}+j \omega \sigma \mu_{0}}$, $g\left(r_{\text {in }}, r_{\text {out }}, \lambda\right)=\int_{\lambda r_{\text {in }}}^{\lambda r_{\text {out }}} x J_{1}(x) d x$, where $J_{1}(x)$ is the Bessel function of the first kind of order one, $\omega$ is the frequency and $\mu_{0}$ is the magnetic constant. Similarly, one can compute the impedance of the coil in air using the formula

$$
Z_{\text {air }}=2 j \omega \pi \mu_{0} \frac{N^{2}}{\left(r_{\text {out }}-r_{\text {in }}\right)^{2}\left(h_{2}-h_{1}\right)^{2}} Q_{1},
$$

where

\footnotetext{
a Corresponding author: andrei.pokatilov@ttu.ee
} 


$$
Q_{1}=\int_{0}^{\infty} \frac{g^{2}\left(r_{\text {in }}, r_{\text {out }}, \lambda\right)}{\lambda^{5}}\left[h_{2}-h_{1}+\frac{e^{-\lambda\left(h_{2}-h_{1}\right)}-1}{\lambda}\right] d \lambda
$$

The function $Q_{1}$ to be integrated has a dominant peak as shown in Figure 1. The width of the peak exhibits inverse dependence on the outer diameter of the coil. The optimization results presented below can be also applied for the sensors with the smaller outer diameters.

In order to save computational costs we have investigated the effect of the value of the upper limit of integration, $b$, on the accuracy of calculation. For this purpose an integration technique based on the trapezoidal rule has been used. As it can be seen from Figure 2 the relative accuracy less than $0.01 \%$ can be achieved at the upper limit of integration $b=5000$. The $b$ can be further reduced to 2500 by using the variable step size.

Figure 1. The integrand of the resistance value of a coil with parameters as given in Table 1 measured in the air at a frequency of $60 \mathrm{kHz}$.

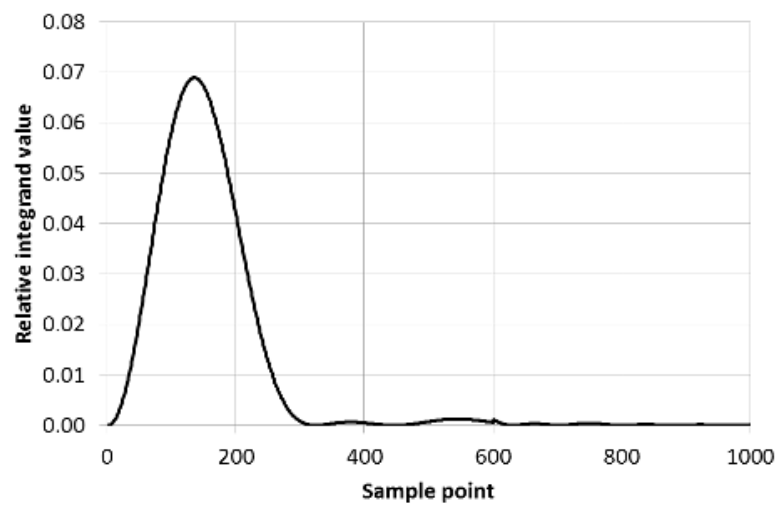

Figure 2. The resistance value deviation against the upper limit of integration of a coil with parameters as given in Table 1 measured in the air at a frequency of $60 \mathrm{kHz}$.

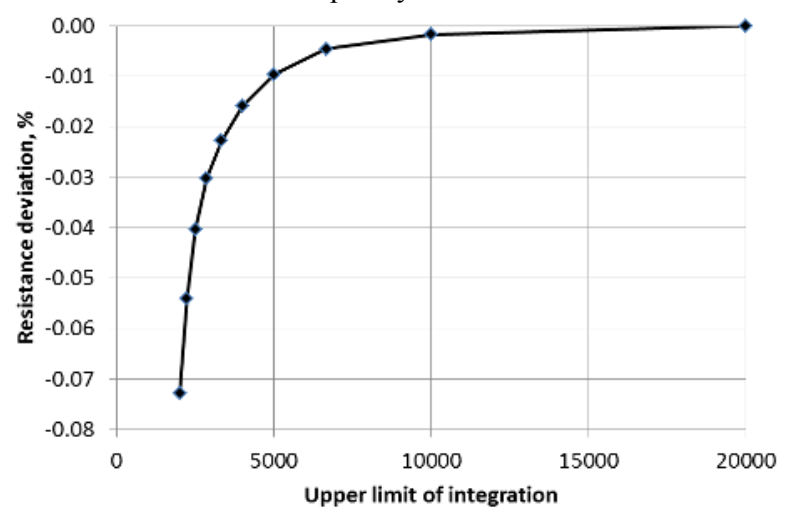

\section{Inverse problem solution}

Equation (1) expresses an impedance value for the known conductivity and lift-off values. In our case the conductivity and lift-off are unknowns to be determined from the impedance measurement by solving the inverse problem. It can be done by minimizing the norm of the difference between theoretical predictions and experimental measurements $[3,5,6]$. However, it has a drawback being trapped at a local minimum.

To find the minimum deviation between theory and experiment we have developed a grid based method which eliminates the risk to be trapped at local minima. Also, the grid-based method has an advantage of visualization of the calculation process at the development stage.

The solution space consisting of the theoretical values of impedance is searched for the best agreement to the experimental result. This is done by application of the point-in-polygon algorithm. The curves forming the grid correspond to the single values of the conductivity and the lift-off.

Two modes of calculation of the grid are proposed: the in-measurement grid and pre-calculated grid.

Figure 3. The search grid after the first iteration and the measurement result (empty circuit) as indicated by the developed software in the high-accuracy mode. Parameters of a coil as given in Table 1 for a metal plate with the conductivity value of $10 \mathrm{MS} / \mathrm{m}$ measured at a frequency of $60 \mathrm{kHz}$.

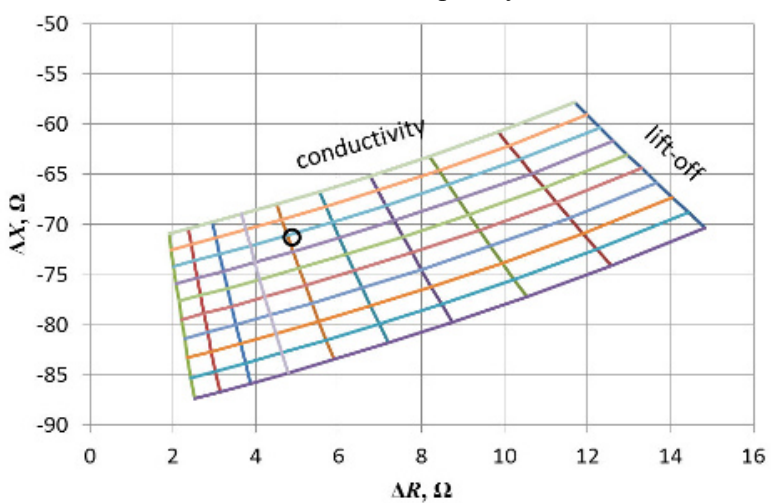

The first approach does not require any preliminary calculations and the search grids are calculated during the measurement, as shown in Figure 3. This allows easier replacement of a sensor and a flexible choice of operating frequencies. For example, for a high-accuracy conductivity measurement the searching space can include the ranges $(1 \ldots 60) \mathrm{MS} / \mathrm{m}$ and $(1 \ldots 1000) \mu \mathrm{m}$ for conductivity and lift-off, respectively. Five iterations of the search grids, narrowing the range of the theoretical values at the each step, are required to determine the conductivity and lift-off values with the computational resolution of less than $0.1 \%$ and $1 \mu \mathrm{m}$, respectively. The computational time for the conductivity value at one frequency is about 25 seconds on the Intel ${ }^{\circledR}$ Core $^{\mathrm{TM}}$ i5 processor.

For less accurate applications where the exact lift-off value is not important and the accuracy level of $1 \%$ for the conductivity value calculation is acceptable the computational time can be reduced. As shown in Figure 4, the search grid includes the lift-off curves approximated by linear functions. This reduces the number of the lift-off curves required by 5 times, keeping the lift-off compensation within the specified searching space. As a result, the computational time is reduced to 5 seconds only.

The computational time can be significantly reduced by forming a pre-calculated grid for the certain sensor at 
a fixed frequency. It requires about $4 \cdot 10^{6}$ and $8 \cdot 10^{3}$ calculated points for the one frequency at the highaccuracy and lift-off approximating modes, respectively.

Figure 4. The search grid after the first iteration and the measurement result (empty circuit) as indicated by the developed software in the lift-off approximating mode. Parameters of a coil as given in Table 1 for a metal plate with the conductivity value of $10 \mathrm{MS} / \mathrm{m}$ measured at a frequency of $60 \mathrm{kHz}$.

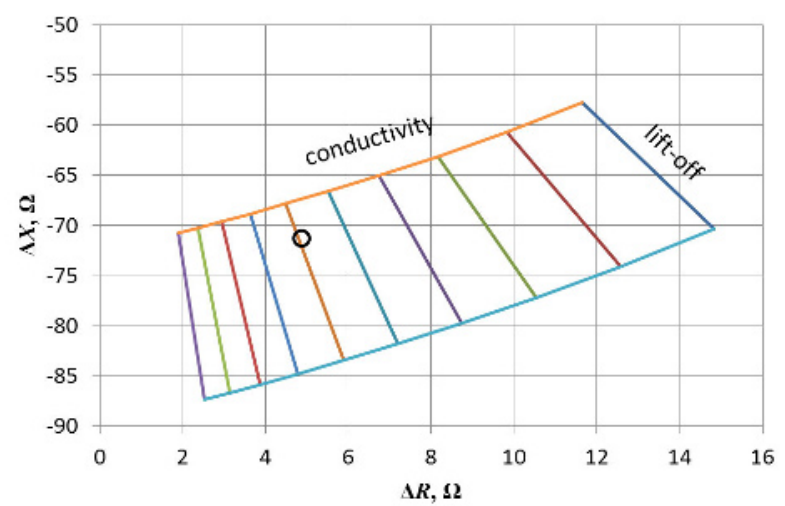

\section{Software description}

The presented method has been realised in LabView ${ }^{\mathrm{TM}}$ and $C++$ programming environments.

LabView $^{\mathrm{TM}}$ and $\mathrm{C}++$ software realization of the forward problem solution demonstrate a good agreement in accuracy of calculation. However, $C++$ code appeared to be about $20 \%$ faster taking about 0.04 seconds per one calculation point on Intel ${ }^{\circledR}$ Core $^{\mathrm{TM}}$ i5 processor.

As the computational time is not critical in the laboratory applications the final solution has been implemented in LabView ${ }^{\mathrm{TM}}$ software having the readyto-use instrument drivers and graphical user interface.

\section{Measurements}

The inductive planar coil used in the AC electrical conductivity measurements has been winded from a commercially available copper wire. The inner and outer radii of the coil and the thickness of the wire have been measured by a measurement microscope. The parameters of the coil as optimized in [7] are summarized in Table 1.

Table 1. Parameters of the coil.

\begin{tabular}{|c|c|}
\hline Parameter & Value \\
\hline inner radius, $r_{\text {in }}$ & $6.66 \mathrm{~mm}$ \\
\hline outer radius, $r_{\text {out }}$ & $24.85 \mathrm{~mm}$ \\
\hline height of the coil, $h$ & $0.15 \mathrm{~mm}$ \\
\hline number of turns, $N$ & 90 \\
\hline
\end{tabular}

The impedance values required for the determination of the electrical conductivity of the object have been measured by an accurate LCR meter over the frequency range from $15 \mathrm{kHz}$ to $480 \mathrm{kHz}$. The measurements were performed inside an air thermostat to keep the temperature stable during the measurements.
The measured electrical conductivity at the AC current has been compared to the electrical conductivity value of approximately $10 \mathrm{MS} / \mathrm{m}$ measured at the DC current by the Van der Pauw method. The deviation of the AC conductivity from the DC conductivity value and the lift-off values are presented in Figure 5.

The measurements required for determination of the $\mathrm{AC}$ and DC conductivity values are traceable to SI units through the national measurement standards.

Figure 5. The deviation of the AC electrical conductivity from the DC conductivity value - diamonds and the lift-off values triangles.

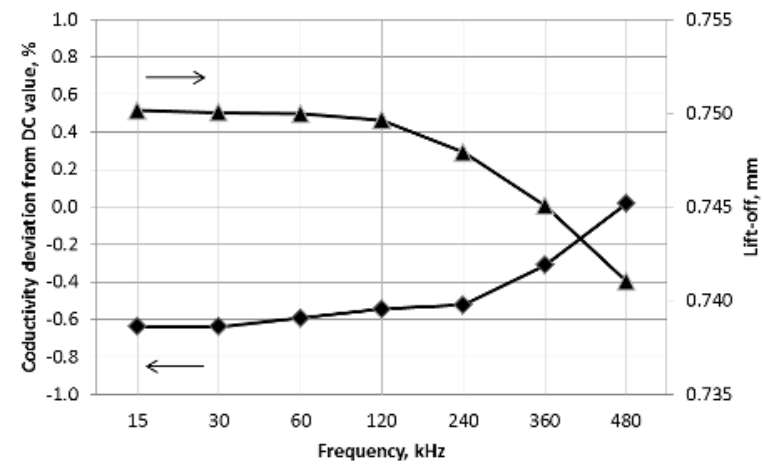

Over the investigated frequency range the conductivity values measured by the $\mathrm{DC}$ and $\mathrm{AC}$ methods are in agreement within $0.7 \%$ of the measured value. The lift-off change is about $10 \mu \mathrm{m}$ from the low frequency values. Frequency dependencies of the conductivity and lift-off values are caused, probably, by the properties of the measurement sensor revealing need for further investigations.

\section{Conclusions}

The method for the accurate computation of the electrical conductivity of non-ferrous metal samples in the frequency range from $15 \mathrm{kHz}$ to $500 \mathrm{kHz}$ has been developed. The proposed technique exploits the theory developed by Dodd and Deeds and the grid-based algorithm.

The forward problem solution realization has been optimized to save the computational costs. The inverse problem solution is based on the search grid for minimization the difference between measured and calculated resistance and reactance of a measurement coil. The grid computation is conducted iteratively: two free parameters, the electrical conductivity and the lift-off are changed to minimize the difference and to obtain the accurate electrical conductivity value. Two specially developed software realizations have been implemented based on the LabView ${ }^{\mathrm{TM}}$ and $C++$ programming environments. In our studies, both software codes have demonstrated accurate determination of the electrical conductivity.

The measured AC and DC conductivity values are in agreement within $0.7 \%$ of the measured value over the frequency range studied. The frequency dependence of 
the AC conductivity and lift-off values is, most probably, due to parameters of the measurement coil used.

\section{Acknowledgments}

The research within the present study has received funding from the Tallinn University of Technology under Grant No BF04. Also, the authors would like to acknowledge the financial support of the Estonian Ministry of Economic Affairs and Communication.

\section{References}

[1] G. Rietveld, Ch. V. Koijmans, L. C. A. Henderson, M. J. Hall, S. Harmon, P. Warnecke, and B. Schumacher, "DC Conductivity Measurements in the Van Der Pauw Geometry," IEEE Trans. Instrum. Meas., 52, pp. 449-453, (2003).

[2] A. C. Lynch, A. E. Drake, C.H. Dix, "Measurement of eddy current conductivity," IEE Proc. A Sci. Meas. Technol., 130, pp. 254-260, (1983).

[3] A. Pokatilov, M. Parker, A. Kolyshkin, O. Märtens, T. Kübarsepp, "Inhomogeneity Correction in Calibration of Electrical Conductivity Standards," Measurement, 46, pp. 1536-1540, (2013).

[4] C. V. Dodd, W. E. Deeds, "Analytical solutions to eddy-current probe-coil problems," Journal of Applied Physics, 39, pp. 2829-2838, (1968).

[5] N. Bowler, Y. Huang, "Electrical conductivity measurement of metal plates using broadband eddycurrent and four point methods," Meas. Sci. Technol., 16, pp. 2193-2200, (2005).

[6] D. J. Harrision, L. D. Jones, S.K. Burke, "Benchmark problems for defect size and shape determination in eddycurrent nondestructive evaluation," Journal of Nondestructive Evaluation, 15, pp. 21-34, (1996).

[7] M. Parker, A. Pokatilov, T. Kübarsepp, O. Märtens, A. Kolyshkin, "Investigation of planar coil for eddy current conductivity measurements in wide frequency range," 19th IMEKO TC-4 Symposium on Measurements of Electrical Quantities, Barcelona, Spain, (2013). 